\title{
Erratum to: Very ample but not normal lattice polytopes
}

\section{Shoetsu Ogata}

\section{Erratum to: Beitr Algebra Geom DOI 10.1007/s13366-011-0077-z}

In the original publication of the article, there was a mistake in the proof of Proposition 1 , because the cone $C\left(P_{q}\right)=\mathbb{R}_{\geq 0}\left(P_{q}-v_{n}\right)$ at the vertex $v_{n}$ is not unimodular for $n \geq 4$. The true statement is the following proposition.

Proposition 1 Let $P_{q}$ be the lattice polytope defined by (5). For $n=3, P_{q}$ is very ample. When $n \geq 4$, if $q \equiv 0,1(\bmod n-2)$, then $k P_{q}$ is very ample for $k \geq 2$.

Proof The set of vertices of $P_{q}$ is $P_{q} \cap M=\left\{v_{0}, \ldots, v_{n}, v_{0}^{+}, \ldots, v_{n}^{+}\right\}$. The affine monoid $C_{v}\left(P_{q}\right) \cap M$ of the cone $C_{v}\left(P_{q}\right)=\mathbb{R}_{\geq 0}\left(P_{q}-v\right)$ at each vertex $v$ except $v_{n}$ of $P_{q}$ is generated by $\left(P_{q}-v\right) \cap M$ as in the original publication.

For the vertex $v_{n}$, set $C\left(P_{q}\right):=\mathbb{R}_{\geq 0}\left(P_{q}-v_{n}\right)$ the simplicial cone with vertex $v_{n}$ at the origin. We have

$$
C\left(P_{q}\right)=\sum_{i=1}^{n-1} \mathbb{R}_{\geq 0}\left(v_{i}-v_{n}\right)+\mathbb{R}_{\geq 0}\left(v_{n}^{+}-v_{n}\right) .
$$

We see that if $n=3$ the cone $C\left(P_{q}\right)$ is unimodular, but if $n \geq 4$ it is not unimodular.

We treat the case when $n \geq 4$.

The online version of the original article can be found under doi:10.1007/s13366-011-0077-z.

\section{S. Ogata $(\varangle)$}

Mathematical Institute, Tohoku University, Sendai 980-8578, Japan

e-mail: ogata@math.tohoku.ac.jp 
When $q \equiv 0(\bmod n-2)$, set

$$
w^{\prime}:=\frac{1}{n-2} \sum_{i=1}^{n-1}\left(v_{i}-v_{n}\right) \in\left(2\left(P_{q}-v_{n}\right)\right) \cap M .
$$

Then we can decompose the face $\sum_{i=1}^{n-1} \mathbb{R}_{\geq 0}\left(v_{i}-v_{n}\right)$ into the union of unimodular cones by the barycentric decomposition at $w^{\prime}$. Thus the semi group $C\left(P_{q}\right) \cap M$ is generated by $\left(2\left(P_{q}-v_{n}\right)\right) \cap M$.

When $q \equiv 1(\bmod n-2)$, set

$$
w:=\frac{1}{n-2}\left(\sum_{i=1}^{n-1}\left(v_{i}-v_{n}\right)+\left(v_{n}^{+}-v_{n}\right)\right) \in\left(2\left(P_{q}-v_{n}\right)\right) \cap M .
$$

Then we can decompose $C\left(P_{q}\right)$ into the union of unimodular cones by the barycentric decomposition at $w$. Thus the semi group $C\left(P_{q}\right) \cap M$ is generated by $\left(2\left(P_{q}-v_{n}\right)\right) \cap M$.

This implies that $k P_{q}$ is very ample for $k \geq 2$.

Remark For any $q$, we can decompose the cone $C\left(P_{q}\right)$ into a union of unimodular cones by using lattice points in $(n-2)\left(P_{q}-v_{n}\right)$. From this and Proposition 3 we see that if $q \geq(2 n-3)(n-2)+1$, then $(n-2) P_{q}$ is very ample but not normal. 